\title{
Lidocaine Stimulates the Function of Natural Killer Cells in Different Experimental Settings
}

\author{
JUAN P. CATA ${ }^{1,2}$, MARIA F. RAMIREZ ${ }^{2,3}$, JOSE F. VELASQUEZ ${ }^{2,4}$, AI DI $^{5}$, KEYURI U. POPAT $^{1}$, \\ VIJAYA GOTTUMUKKALA ${ }^{1,2}$, DAHLIA M. BLACK ${ }^{6}$, VALERAE O. LEWIS $^{7}$ and JEAN N. VAUTHEY ${ }^{8}$ \\ Departments of ${ }^{1}$ Anesthesiology and Perioperative Medicine, ${ }^{6}$ Breast Surgical Oncology, \\ ${ }^{7}$ Orthopedic Oncology, and ${ }^{8}$ Surgical Oncology, \\ The University of Texas MD Anderson Cancer Center, Houston, TX, U.S.A.; \\ ${ }^{2}$ Anesthesiology and Surgical Oncology Research Group, Houston, TX, U.S.A.; \\ ${ }^{3}$ Department of Anesthesiology, Massachusetts General Hospital, Boston, MA, U.S.A.; \\ ${ }^{4}$ University CES, School of Medicine, Medellin, Colombia; \\ ${ }^{5}$ Department of Pathology, Texas A\&M Cancer Center, Baylor Scott \& White Memorial Hospital, Temple, TX, U.S.A.
}

\begin{abstract}
Background: One of the functions of natural killer (NK) cells is to eliminate cancer cells. The cytolytic activity of NK cells is tightly regulated by inhibitory and activation receptors located in the surface membrane. Lidocaine stimulates the function of $\mathrm{NK}$ cells at clinically relevant concentrations. It remains unknown whether this effect of lidocaine has an impact on the expression of surface receptors of NK cells, can uniformly stimulate across different cancer cell lines, and enhances the function of cells obtained during oncological surgery. Materials and Methods: NK cells from healthy donors and 43 patients who had undergone surgery for cancer were isolated. The function of NK cells was measured by lactate dehydrogenase release assay. NK cells were incubated with clinically relevant concentrations of lidocaine. By flow cytometry, we determined the impact of lidocaine on the expression of galactosylgalactosylxylosylprotein3-betaglucuronosytranferase 1, marker of cell maturation (CD57), killer cell lectin like receptor $A$, inhibitory $(N K G 2 A)$ receptors and killer cell lectin like receptor $D$, activation (NKG2D) receptors of $N K$ cells. Differences in expression at $p<0.05$ were considered statistically significant. Results: Lidocaine increased
\end{abstract}

This article is freely accessible online.

Correspondence to: Juan P. Cata, MD, Department of Anesthesiology and Perioperative Medicine, The University of Texas MD Anderson Cancer Center, 1515 Holcombe Blvd, Unit 409, Houston, TX 77005, U.S.A. Tel: +1 7137924582, Fax: +1 7137924582, email: jcata@mdanderson.org

Key Words: Lidocaine, natural killer cells, cytotoxicity, cancer cells. the expression of $N K G 2 D$ receptors and stimulated the function of NK cells against ovarian, pancreatic and ovarian cancer cell lines. Lidocaine also increased the cytolytic activity of $\mathrm{NK}$ cells from patients who underwent oncological surgery, except for those who had orthopedic procedures. Conclusion: Lidocaine showed an important stimulatory activity on NK cells. Our findings suggest that lidocaine might be used perioperatively to minimize the impact of surgery on NK cells.

Inflammation and immunosuppression are hallmark phenomena of oncological surgery $(1,2)$. Animal studies indicate that the inflammatory response and suppression of the innate immunity observed during and after surgery favor the formation of metastases $(3,4)$. Human studies demonstrate that a high postoperative inflammatory state is associated with shorter survival due to recurrence or tumor progression $(5,6)$. Different investigations also have shown that the function and count of natural killer (NK) cells decrease during and after surgery and correlates with an increase in locoregional and distant metastasis (7). Therefore, a significant emphasis has been placed on the identification of perioperative interventions that can modulate the function of NK cells (8).

Lidocaine, an amide local anesthetic, has strong antiinflammatory effects when used during and after surgery (9). Lidocaine also modulates the immune system. While at high concentrations lidocaine can suppress the function of NK cells, it stimulates the killing activity of these cells at therapeutic plasma concentrations $(10,11)$. Furthermore, the stimulating effect of lidocaine on NK cells appears not to be target cell-specific. Briefly, our group recently demonstrated that lidocaine enhanced the killing activity of NK cells against three different leukemia cell lines (11). Based on these 
observations, it is possible to speculate that lidocaine effectively increases the cytotoxic effect of NK cells across several tumor types. However, to date, as far as we are aware, there is no evidence to conclude that lidocaine stimulates the activity of human NK cells against cells lines from solid tumors. Furthermore, all studies using lidocaine have used NK cells from healthy donors or non-cancer patients. Thus, it remains unknown whether lidocaine can restore the function of cells from patients undergoing cancer surgery.

In the present laboratory study, we tested the following two hypotheses. Firstly, if lidocaine increases the killing activity of NK cells by modulating the expression of maturation, inhibitory and activating receptors. Secondly, if lidocaine stimulates not only the function of NK cells from healthy donors against cell lines from solid malignancies but also the cytotoxic activity of NK cells isolated from patients who underwent cancer surgery.

\section{Materials and Methods}

We received an Institutional Review Board (\#2011-0146, \#PA120508 and \#PA12-0524) approval for this study and obtained written informed consent from all patients. All patients underwent volatile general anesthesia with intraoperative and postoperative opioidbased analgesia $(1,2)$. Blood draws occurred before induction of anesthesia, on postoperative days 1, 3 and 5 in patients undergoing bone tumor surgeries and liver resections, and on the morning after surgery in women who underwent mastectomies $(1,2)$.

Cell culture and NK cell isolation. The human leukemia cell line K562 (American Type Culture Collection, Manassas, VA, USA), the osteosarcoma cell line KRIB (kindly donated by Dr. Valeare Lewis), the ovarian cancer cell line SKOV3 (kindly donated by Dr. Anil Sood) and the pancreatic cancer cell line (kindly donated by Dr. Jason Fleming) were cultured in RPMI-1640 medium (SigmaAldrich, St Louis, MO, USA) and supplemented with $10 \%$ or $20 \%$ fetal bovine serum, $1 \%$ penicillin-streptomycin, and $1 \% \mathrm{~L}-$ glutamine (all from Gibco Life Technologies, Carlsbad, CA, USA) (11). NK cells were isolated from either buffy coats (peripheral blood mononuclear cells, PBMCs) from de-identified healthy blood donors or from patients who underwent breast cancer, liver resection or malignant bone surgeries at MD Anderson Cancer Center as published elsewhere $(1,2,11)$.

NK cells from donors and patients were isolated by positive selection using magnetic cell separation (Miltenyi Biotec, Bergicsh Gladbach, Germany), and NK cell viability was tested with trypan blue. Samples with more than $95 \%$ viability were used in the cytotoxicity assays $(1,11)$.

Flow cytometric studies. We investigated the impact of lidocaine on NK cell-surface marker of differentiation, activation, and inhibition. NK cells isolated from healthy donors were incubated with lidocaine (vehicle: sodium chloride $0.9 \%, 0.01,0.1,1,10$, and $50 \mu \mathrm{M}$ ) for 20 $\mathrm{h}$ (11). Determination of expression of activating and inhibitory receptors was conducted by incubation of NK cells with anti-CD57 anti-NKG2A and anti-NKG2D and respective isotype controls (all from Becton Dickinson, Franklin Lakes, NJ, USA), as recommended by the manufacturer. Washing was performed with washing buffer
PBS-F. After final washing, cell-associated fluorescence was determined by flow cytometry after appropriate gating.

Cytotoxicity assays. NK cell cytotoxicity was calculated using the lactate dehydrogenase (LDH) assay (Cytotoxicity Detection Kit, 630117; Clontech Laboratories, Mountain View, CA, USA) (1, 11). This technique measures the release of $\mathrm{LDH}$ from dying cells in the culture medium. To test the effect of increasing concentrations of lidocaine (vehicle, $0.01,0.1,1,10$, and $50 \mu \mathrm{M}$ ) on the NK cell cytotoxicity against target cells (SKOV3, KRIB, and PATC53 cells), we incubated effector (NK cells) and target cells for $20 \mathrm{~h}(1,11)$. Each assay was repeated a minimum of five times. To test the effect of lidocaine $(0.01 \mu \mathrm{M})$ on the cytotoxic activity of $\mathrm{NK}$ cells obtained from patients who underwent surgery, we incubated the effectors and target cells (K562 cells) for 20 hours. The percentage of cytotoxicity was calculated according to the following equation:

Cytotoxicity \%=[(effector: target cell mix - effector cell control $)-$ low control]/(high control - low control) $\times 100$.

Each experiment was performed in triplicate $(1,11)$.

Statistical analysis. The results from cytotoxicity assays are reported in means and standard deviations or medians and $25-75 \%$ percentiles according to normality test (D'Agostino and Pearson omnibus test). Statistical significance for NK cell cytotoxicity assays performed to test the effect of different concentrations of lidocaine against HepG2, SKOV3, KRIB and MDA-PATC53 cells was established by Kruskal-Wallis test followed by Dunn test for multiple comparisons when appropriate. Wilcoxon matched-pairs signed ranked test was conducted to evaluate the effect of a single dose of lidocaine on $\mathrm{NK}$ cell cytotoxicity from patients. We considered that an increase of NK cell cytotoxicity by at least $50 \%$ on postoperative day 1 would be clinically relevant. Based on previously published studies, we assumed a mean postoperative NK cell cytotoxicity of $10 \%$ and a standard deviation of $8 \%$. Therefore, we would need at least 41 patients to detect a minimum increase of $50 \%$ in the NK cell cytotoxicity function of cells treated with lidocaine compared with vehicle-treated cells. Values of $p<0.05$ were considered statistically significant. Prism 5 (GraphPad Software, Inc, San Diego, CA, USA) was used to perform all statistical analysis.

\section{Results}

Effect of lidocaine on surface markers of maturation, activation, and inhibition. We have previously shown that lidocaine appears to increase the killing activity of NK cells by a direct action on these cells (11). To further investigate our previous findings, we measured the effect of lidocaine on the expression of three surface markers involved in cell differentiation, activation, and inhibition. As shown in Table I and Figure 1, lidocaine caused a small but not statistically significant increase in the expression of CD57 (indicative of NK cell differentiation). Lidocaine did not have any impact on the mean intensity fluorescence of the NKG2A receptor (inhibitory receptor). However, the flow cytometric analysis 

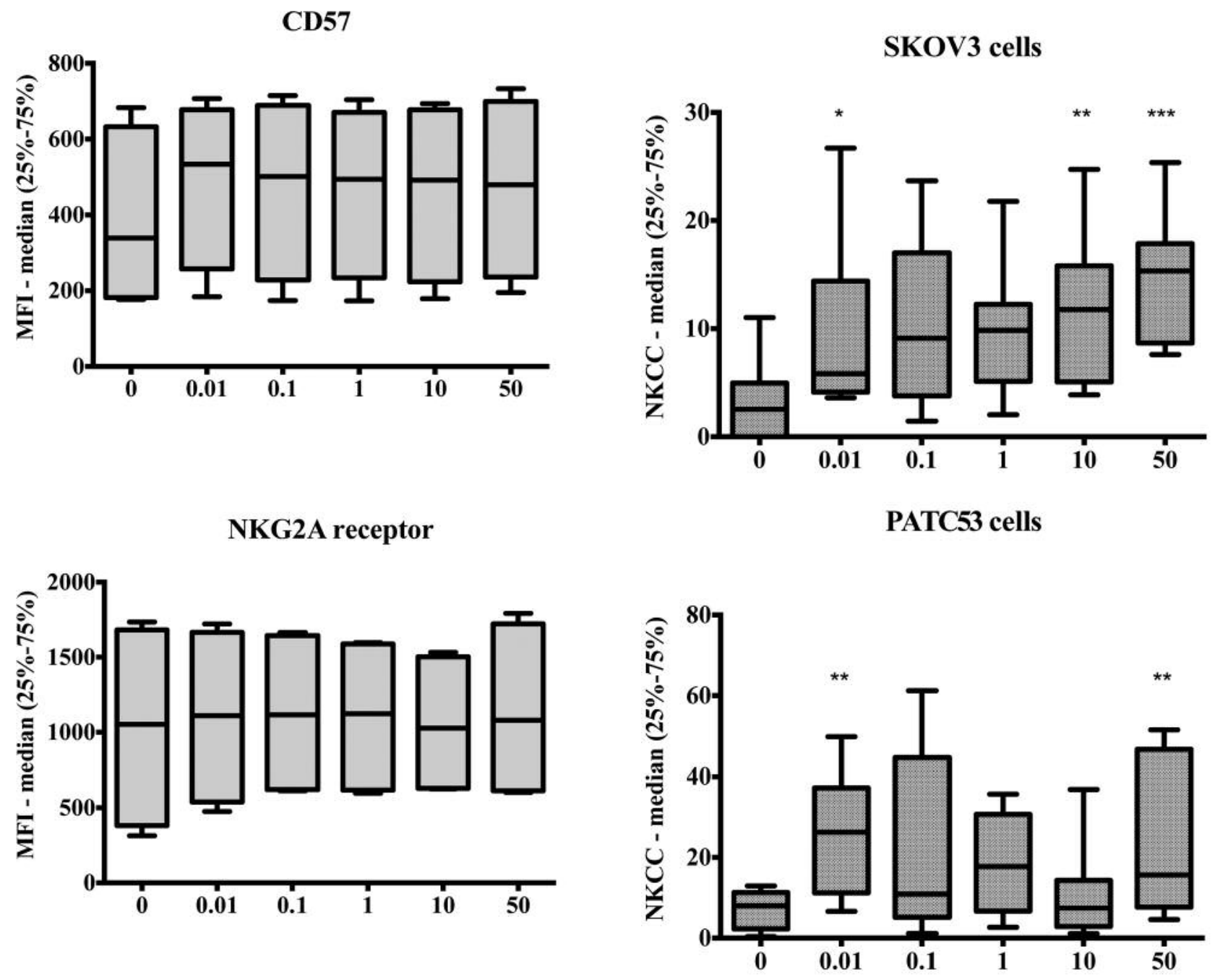

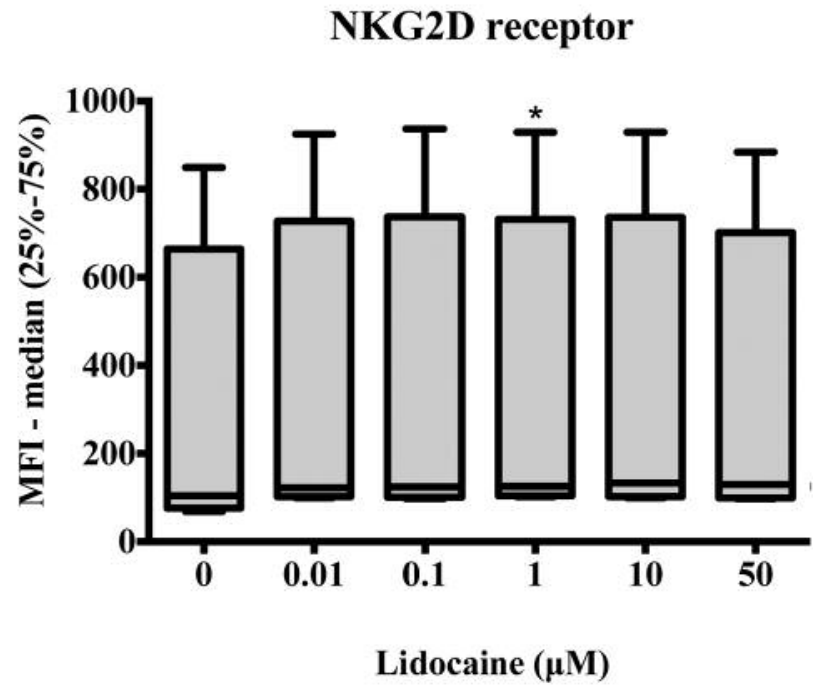

Figure 1. Effect of lidocaine on the expression of surface markers of maturation, inhibition and activation of NK cells.
SKOV3 cells

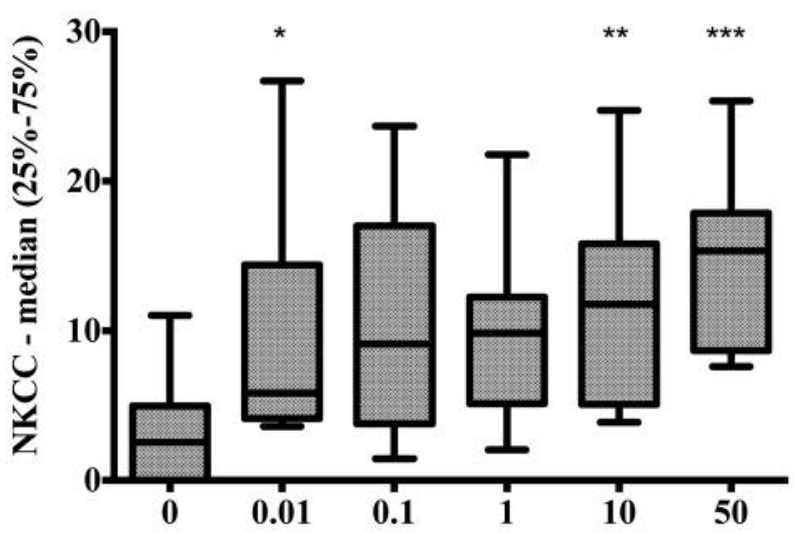

PATC53 cells

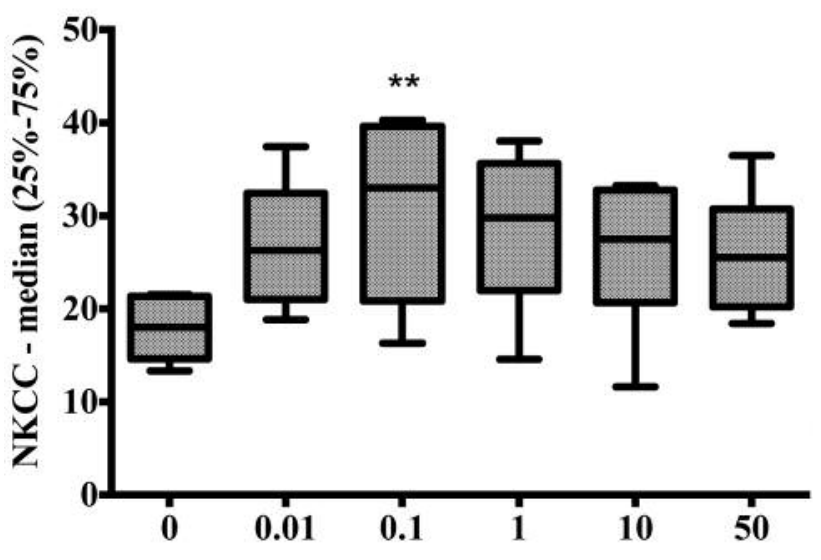

Figure 2. Effect of lidocaine on the NK cell activity against ovarian, pancreatic and osteosarcoma cell lines. 
Table I. Effect of lidocaine on the expression of surface markers of maturation, inhibition and activation of natural killer cells.

\begin{tabular}{|c|c|c|c|c|c|c|c|}
\hline \multirow[t]{2}{*}{ Marker } & \multicolumn{6}{|c|}{ Lidocaine concentration $\mu \mathrm{M}$} & \multirow{2}{*}{$\begin{array}{c}p \text {-Value } \\
\text { Overall } \\
\text { effect }\end{array}$} \\
\hline & 0 & 0.01 & 0.1 & 1 & 10 & 50 & \\
\hline CD57 & $339(181.8-632.8)$ & $534(257.5-677.8)$ & $501(228.3-689.3)$ & 494 (234-670.8) & $492(223.5-492)$ & $479.5(236-699.8)$ & 0.13 \\
\hline NKG2A & $1053(381.5-1682)$ & $1111(538-1666)$ & $1117(621.5-1645)$ & $1123(618.3-1589)$ & $1028(629.3-1503)$ & $1080(613.3-1723)$ & 0.78 \\
\hline NKG2D & $104.1(76.0-664)$ & $122(102.4-727.3)$ & $124(101.1-737)$ & $125.5 *(104.3-731.8)$ & $133(102.1-736)$ & $130(99.4-701.5)$ & 0.05 \\
\hline
\end{tabular}

CD57: Marker of maturation; NKG2A: inhibitory receptor; NKG2D: activating receptor. ${ }^{*} p<0.05$ compared to $0 \mu \mathrm{M}$ (vehicle). Data are shown as median (25-75\% percentile).

Table II. Effect of lidocaine on natural killer cell activity against ovarian, pancreatic and osteosarcoma cell lines.

\begin{tabular}{|c|c|c|c|c|c|c|c|}
\hline \multirow[t]{2}{*}{ Cell line } & \multicolumn{6}{|c|}{ Lidocaine concentration $\mu \mathrm{M}$} & \multirow{2}{*}{$\begin{array}{c}p \text {-Value } \\
\text { Overall } \\
\text { effect }\end{array}$} \\
\hline & 0 & 0.01 & 0.1 & 1 & 10 & 50 & \\
\hline SKOV3 & $2.54(0.01-4.95)$ & $5.83 *(4.13-14.4)$ & $9.1(3.78-17.01$ & $9.82(5.11-12.24)$ & $11.76^{* *}(5.07-15.82)$ & $15.34 * * *(8.67-17.85)$ & ) 0.0003 \\
\hline PATC53 & $8(2.24-11.3)$ & $26.24(11.2-37.21)$ & $10.9(5.13-44.63)$ & $17.23(6.67-30.68)$ & $7.43(2.83-14.29)$ & $15.64(7.66-46.68)$ & 0.003 \\
\hline KRIB & $18.01(14.6-21.34)$ & $26.29(21.01-32.43)$ & $33.01 * *(20.83-39.62)$ & $29.79(21.97-35.63)$ & $27.5(20.65-32.76)$ & $25.5(20.2-30.72)$ & 0.04 \\
\hline
\end{tabular}

SKOV3: Ovarian cancer cells; PATC53: pancreatic cancer cells; KRIB: osteosarcoma cells. Significantly different from the vehicle-treated control $(0 \mu \mathrm{M})$ at: ${ }^{*} p<0.05,{ }^{*} p<0.01$ and ${ }^{* * *} p<0.005$. Data are shown as median $(25-75 \%$ percentile $)$.

indicated that lidocaine caused a slight, but borderline statistically significant increase in the expression of activating receptor $\mathrm{NKG} 2 \mathrm{D}$.

Effect of lidocaine on cytotoxicity of NK cells from healthy donors against target cells from patients with solid cancer. As shown in Figure 2, lidocaine significantly increased the cytotoxicity function of NK cells against all targets cells. SKOV3 cells showed the highest sensitivity to NK cellkilling activity across all concentrations of lidocaine. Furthermore, the concentration at which this local anesthetic had the largest effect was not the same among all cell lines. Briefly, $50 \mu \mathrm{M}$ of lidocaine caused a 7-fold increase in the killing activity of NK cells against SKOV3 cells. On the contrary, we observed that lower concentrations of lidocaine had the strongest effects on PATC53 and KRIB cells (Figure 2 and Table II).

Lidocaine stimulates the function of $N K$ cells from patients with cancer. We tested the effect of lidocaine on NK cells from 43 patients who underwent mastectomies, liver resections or orthopedic oncological surgery. In comparison to the vehicle, lidocaine increased by two-fold the function of NK cells obtained before surgery (Table III). A more significant effect was observed when lidocaine was added to NK cells isolated postoperatively (Table III). Briefly, the cytotoxic activity of NK cells obtained on postoperative day 1,3 and 5 that were treated with lidocaine was 4, 5.7 and 4 times higher than those incubated with vehicle, respectively. We then assessed if the observed effects of lidocaine were surgery specific. Thus, we analyzed the in vitro effect of lidocaine on the function NK cells obtained from 15 women who had undergone mastectomies (Table III). Lidocaine $(0.01 \mu \mathrm{M})$ stimulated the in vitro killing activity of the NK cells, not only of those collected preoperatively but also of those obtained after surgery (Table III). We observed a 2-fold and nearly 4-fold increase in the cytotoxic activity of NK cells isolated preoperatively and postoperatively, respectively.

Similarly, we co-treated target (K562) cells and NK cells obtained from 24 patients undergoing liver resections with lidocaine at $0.01 \mu \mathrm{M}$ (Table III). As shown in Figure 2, NK cells isolated preoperatively and treated with lidocaine showed a 2-fold increase in their killing activity compared to those treated with the medium. Moreover, lidocaine stimulated the function of NK cells obtained on postoperative day 1,3 and 5 by $321 \%, 349 \%$, and $625 \%$, respectively (Table III).

Lastly, we tested the effect of lidocaine on NK cells isolated from four patients with bone malignancies. Although we observed an increase in the function of NK cells treated with lidocaine, the impact was not statistically significant (Table III). 
Table III. In vitro effect of lidocaine on the natural killer cell function of patients undergoing surgery.

\begin{tabular}{|c|c|c|c|c|c|c|c|c|c|c|c|}
\hline \multirow{2}{*}{$\begin{array}{l}\text { Time } \\
\text { point }\end{array}$} & \multicolumn{2}{|c|}{ All patients $(n=43)$} & \multirow[t]{2}{*}{$p$-Value } & \multicolumn{2}{|c|}{ Mastectomy $(\mathrm{n}=15)$} & \multirow[t]{2}{*}{$p$-Value } & \multicolumn{2}{|c|}{ Liver resection $(\mathrm{n}=24)$} & \multirow[t]{2}{*}{$p$-Value } & \multicolumn{2}{|c|}{ Orthopedic surgery $(\mathrm{n}=4) p$-Value } \\
\hline & Vehicle & Lidocaine & & Vehicle & Lidocaine & & Vehicle & Lidocaine & & Vehicle & Lidocaine \\
\hline Preop & $\begin{array}{l}22.86(16.42- \\
35.92)\end{array}$ & $\begin{array}{c}44.63(28.98- \\
61.06)\end{array}$ & $<0.0001$ & $\begin{array}{c}28.9(18.2- \\
39.29)\end{array}$ & $\begin{array}{c}63.6(42.76- \\
77.39)\end{array}$ & 0.001 & $\begin{array}{l}21.68(14.4- \\
29.77)\end{array}$ & $\begin{array}{l}40.82(26.1- \\
53.25)\end{array}$ & 0.002 & $\begin{array}{l}25.81(16.41- \\
29.19)\end{array}$ & $\begin{array}{c}31.15(31.15-0.25 \\
146.4)\end{array}$ \\
\hline POD1 & $\begin{array}{c}4.75 \\
(1.39-16.7)\end{array}$ & $\begin{array}{c}18.67(9.91- \\
34.33)\end{array}$ & $<0.0001$ & $\begin{array}{c}3.56(0.4- \\
17.23)\end{array}$ & $\begin{array}{l}12.06(5.16- \\
28.17)\end{array}$ & 0.024 & $\begin{array}{c}4.66(1.93- \\
14.83)\end{array}$ & $\begin{array}{c}19.66(10.4- \\
39.02)\end{array}$ & $<0.0001$ & $\begin{array}{l}12.35(3.28- \\
19.74)\end{array}$ & $\begin{array}{l}27.63(16.81-0.125 \\
34.04)\end{array}$ \\
\hline POD3 & $\begin{array}{c}3.78(0.24- \\
16.13)\end{array}$ & $\begin{array}{c}21.44(10.45- \\
29.36)\end{array}$ & 0.0002 & NA & NA & & $\begin{array}{c}4.43(0.57- \\
16.13)\end{array}$ & $\begin{array}{l}19.92(8.76- \\
29.36)\end{array}$ & 0.002 & $\begin{array}{l}1.81(0- \\
24.75)\end{array}$ & $\begin{array}{c}23.24(18.94-0.125 \\
46.63)\end{array}$ \\
\hline POD5 & $\begin{array}{c}5.31(0.82- \\
12.81)\end{array}$ & $\begin{array}{c}21.24(12.42 \\
-37.86\end{array}$ & $<0.0001$ & NA & NA & & $\begin{array}{c}2.86(0- \\
12.81)\end{array}$ & $\begin{array}{c}20.79(7.24- \\
39.29)\end{array}$ & 0.001 & $\begin{array}{c}6.5(2.93- \\
14.48)\end{array}$ & $\begin{array}{l}22.72(18.44-0.125 \\
40.98)\end{array}$ \\
\hline
\end{tabular}

NA: Blood specimens not available; Preop: preoperative; POD: postoperative day. Data are shown as median (25-75\% percentile).

\section{Discussion}

Previous studies have shown that cell lines from different solid tumors are sensitive to the killing activity of NK cells (12-14). However, we demonstrated, to our knowledge for the first time, that lidocaine in vitro has strong stimulatory effects on the killing activity of NK cells against pancreatic, ovarian and osteosarcoma cells. Our results are in agreement with previously reported findings from our group in which we showed that lidocaine stimulated the function of NK cells against three different leukemia cell lines (11).

In that study, we demonstrated that the effect of lidocaine is primarily due to its action on NK cells (11). In the present work, we have tried to explore the mechanisms of our findings further. We found that lidocaine induced a small increase in the expression of the activating receptor NKG2D but had no significant effects on the expression of markers of maturation or the inhibitory receptor NKG2A. Whether a small increase in the expression of the NKG2D receptor is solely responsible for the observed strong effect of lidocaine on $\mathrm{NK}$ cell cytotoxicity remains unknown. However, it is worth considering that lidocaine also acts on cancer cells, which can also, at least in part, explain our results (11). Piegeler et al. recently reported that lidocaine blocks proto-oncogene tyrosine-protein kinase (Src) and protein kinase B (Akt) phosphorylation in lung cancer and endothelial cells (15-17). A recent study indicated that cancer cells treated with sunitinib, a multitargeted tyrosine kinase inhibitor, showed increased sensitivity against NK cells (18). Furthermore, lidocaine was found to bind platelet activation factor (PAF) receptor (19). Berthou et al. observed that PAF activation made NK cellresistant cancer cells susceptible to the lytic activity of NK cells (20). Thus, it is possible to speculate that lidocaine might also sensitize cancer cells to the effect of NK cells by acting as tyrosine kinase inhibitor or binding the PAF receptor.

Our findings are supported by a previous study that demonstrated that NK cells from patients who received an intravenous infusion of lidocaine were more cytotoxic than those from patients who had epidural anesthesia or placebo (21). Similarly, Yokama et al. demonstrated that the administration of lidocaine for epidural anesthesia did not have any effect on the function of NK cells in comparison to placebo (22). In the present study, we also demonstrated that lidocaine in vitro and clinically relevant concentrations significantly stimulated NK cell cytotoxicity in the context of breast cancer surgery and liver resections. Although we did not show the same effect of lidocaine on NK cells obtained from patients undergoing orthopedic oncological surgery, we could speculate that our results were due to the small sample size $(n=4)$ since the data indicate that lidocaine-treated cells had a higher activity than vehicle-treated cells. Thus, the literature and our data suggest that systemic lidocaine infusions during and after surgery could be used to preserve the function of NK cells.

Our study has several limitations. Firstly, all experiments were conducted in vitro. Therefore, we do not know if the effects of lidocaine are reproducible in vivo. Secondly, we only explored the effect of lidocaine on three surface markers of NK cells. We took this approach because of the importance of CD57, NKG2DA and NKG2D in the function of NK cells. However, it is possible that lidocaine might affect the expression of other activating or inhibitory receptors. Thirdly, we only tested the effect of lidocaine in three different cell lines and three surgical settings. Hence, our results may not necessarily translate to other experimental systems. Lastly, the pool of available NK cells from patients was limited. Hence, we were unable to explore whether similar mechanisms of stimulation of NK cells from healthy donors can be translated to patients undergoing surgery, or whether higher concentrations of lidocaine lead to stronger effects on NK cell cytotoxicity. Furthermore, we did not test the function of NK cells from patients against their own primary cancer cells.

In conclusion, lidocaine in vitro increases the expression of the NKG2D receptor in NK cells, stimulates the function of these cells against cell lines from solid malignancies, and 
augments NK cell cytotoxicity in the context of oncological surgery. More research is needed to identify the mechanisms behind our findings and investigate whether our results can be translated into the clinical setting.

\section{Acknowledgements}

The Authors acknowledge Drs. A. Sood and J. Fleming for generously donating the SKOV3 and PATC53 cells lines. We acknowledge Dr. Luis Velez for his support with the flowcytometry studies.

\section{References}

1 Ramirez MF, Ai D, Bauer M, Vauthey JN, Gottumukkala V, Kee S, Shon D, Truty M, Kuerer HM, Kurz A, Hernandez M and Cata JP: Innate immune function after breast, lung, and colorectal cancer surgery. J Surg Res 194: 185-193, 2015.

2 Velasquez JF, Ramirez MF, Ai DI, Lewis V and Cata JP: Impaired immune function in patients undergoing surgery for bone cancer. Anticancer Res 35: 5461-5466, 2015.

3 Sorski L, Melamed R, Matzner P, Lavon H, Shaashua L, Rosenne E and Ben-Eliyahu S: Reducing liver metastases of colon cancer in the context of extensive and minor surgeries through beta-adrenoceptors blockade and COX2 inhibition. Brain Behav Immun 58: 91-98, 2016.

4 Matzner P, Sorski L, Shaashua L, Elbaz E, Lavon H, Melamed R, Rosenne E, Gotlieb N, Benbenishty A, Reed SG and BenEliyahu S: Perioperative treatment with the new synthetic TLR4 agonist GLA-SE reduces cancer metastasis without adverse effects. Int J Cancer 138: 1754-1764, 2016.

5 Forget P, Machiels J-P, Coulie P, Berliere M, Poncelet A, Tombal B, Stainier A, Legrand C, Canon J-L, Kremer Y and De Kock M: Neutrophil: Lymphocyte ratio and intraoperative use of ketorolac or diclofenac are prognostic factors in different cohorts of patients undergoing breast, lung, and kidney cancer surgery. Ann Surg Oncol Suppl 3: S650-660, 2013.

6 Lee BM, Rodriguez A, Mena G, Gottumukkala V, Mehran RJ, Rice DC, Feng L, Yu J and Cata JP: Platelet-to-lymphocyte ratio and use of NSAIDs During the perioperative period as prognostic indicators in patients with NSCLC undergoing surgery. Cancer Control 23: 284-294, 2016.

7 Melamed R, Bar-Yosef S, Shakhar G, Shakhar K and BenEliyahu S: Suppression of natural killer cell activity and promotion of tumor metastasis by ketamine, thiopental, and halothane, but not by propofol: mediating mechanisms and prophylactic measures. Anesth Analg 97: 1331-1339, 2003.

8 Horowitz M, Neeman E, Sharon E and Ben-Eliyahu S: Exploiting the critical perioperative period to improve long-term cancer outcomes. Nat Rev Clin Oncol 12: 213-226, 2015.

9 Yardeni IZ, Beilin B, Mayburd E, Levinson Y and Bessler H: The effect of perioperative intravenous lidocaine on postoperative pain and immune function. Anesth Analg 109: 1464-1469, 2009.

10 Krog J, Hokland M, Ahlburg P, Parner E and Tonnesen E: Lipid solubility- and concentration-dependent attenuation of in vitro natural killer cell cytotoxicity by local anesthetics. Acta Anaesthesiologica Scandinavica 46: 875-881, 2002.

11 Ramirez MF, Tran P and Cata JP: The effect of clinically therapeutic plasma concentrations of lidocaine on natural killer cell cytotoxicity. Reg Anesth Pain Med 40: 43-48, 2015.
12 Tallerico R, Todaro M, Di Franco S, Maccalli C, Garofalo C, Sottile R, Palmieri C, Tirinato L, Pangigadde PN, La Rocca R, Mandelboim O, Stassi G, Di Fabrizio E, Parmiani G, Moretta A, Dieli F, Karre K and Carbone E: Human NK cells selective targeting of colon cancer-initiating cells: a role for natural cytotoxicity receptors and MHC class I molecules. J Immunol 190: 2381-2390, 2013.

13 Jewett A, Tseng HC, Arasteh A, Saadat S, Christensen RE and Cacalano NA: Natural killer cells preferentially target cancer stem cells; role of monocytes in protection against NK cell mediated lysis of cancer stem cells. Curr Drug Deliv 9: 5-16, 2012.

14 Castriconi R, Daga A, Dondero A, Zona G, Poliani PL, Melotti A, Griffero F, Marubbi D, Spaziante R, Bellora F, Moretta L, Moretta A, Corte G and Bottino C: NK cells recognize and kill human glioblastoma cells with stem cell-like properties. J Immunol 182: 3530-3539, 2009.

15 Piegeler T, Schlapfer M, Dull RO, Schwartz DE, Borgeat A, Minshall RD and Beck-Schimmer B: Clinically relevant concentrations of lidocaine and ropivacaine inhibit TNFalphainduced invasion of lung adenocarcinoma cells in vitro by blocking the activation of Akt and focal adhesion kinase. Br J Anaesth 115: 784-791, 2015.

16 Piegeler T, Votta-Velis EG, Bakhshi FR, Mao M, Carnegie G, Bonini MG, Schwartz DE, Borgeat A, Beck-Schimmer B and Minshall RD: Endothelial barrier protection by local anesthetics: ropivacaine and lidocaine block tumor necrosis factor-alphainduced endothelial cell Src activation. Anesthesiology 120: 1414-1428, 2014.

17 Piegeler T, Votta-Velis EG, Liu G, Place AT, Schwartz DE, Beck-Schimmer B, Minshall RD and Borgeat A: Antimetastatic potential of amide-linked local anesthetics: inhibition of lung adenocarcinoma cell migration and inflammatory Src signaling independent of sodium channel blockade. Anesthesiology 117: 548-559, 2012.

18 Huang YX, Chen XT, Guo KY, Li YH, Wu BY, Song CY and He YJ: Sunitinib induces NK-kappaB-dependent NKG2D ligand expression in nasopharyngeal carcinoma and hepatoma cells. $\mathrm{J}$ Immunother 40: 164-174, 2017.

19 Hollmann MW, Gross A, Jelacin N and Durieux ME: Local anesthetic effects on priming and activation of human neutrophils. Anesthesiology 95: 113-122, 2001.

20 Berthou C, Bourge JF, Zhang Y, Soulie A, Geromin D, Denizot Y, Sigaux $\mathrm{F}$ and Sasportes M: Interferon-gamma-induced membrane PAF-receptor expression confers tumor cell susceptibility to NK perforin-dependent lysis. Blood 95: 2329-2336, 2000.

21 Yokoyama M, Itano Y, Katayama H, Morimatsu H, Takeda Y, Takahashi T, Nagano O and Morita K: The effects of continuous epidural anesthesia and analgesia on stress response and immune function in patients undergoing radical esophagectomy. Anesth Analg 101: 1521-1527, 2005.

22 Yokoyama M, Itano Y, Mizobuchi S, Nakatsuka H, Kaku R, Takashima T and Hirakawa M: The effects of epidural block on the distribution of lymphocyte subsets and natural-killer cell activity in patients with and without pain. Anesthes Analg 92: 463-469, 2001. 